\title{
Echo simulation of dual polarization Doppler weather radar based on the physical model
}

\author{
Qutie JieLa, Haijiang Wang ${ }^{*}$ D, Shipeng Hu, Jiahui Zhu and Mengqing Gao
}

\footnotetext{
* Correspondence: whj@cuit.edu.cn College of Electronic Engineering, Chengdu University of Information Technology, Chengdu 610225, Sichuan, China
}

\begin{abstract}
Using the scattering characteristics of particles to simulate the radar echo can supply the test signals close to the real precipitation echo for the weather radar and save the time and cost of the research and development and maintenance of the weather radar. In this paper, the precipitation echo of weather radar is simulated based on the theoretical basis that the falling raindrops have a shape well approximated by an oblate spheroid in the atmosphere. The Marshal-Palmer distribution is applied to describe the raindrop spectrum distribution of precipitation particles. It is assumed that the raindrop particles of different sizes have the random distribution in the radar resolution volume, and then the spatial distribution of precipitation particles in the resolution volume is modeled. The echo signals of horizontal and vertical polarization channels of dualpolarization weather radar are obtained by vector superposition of backscattering echoes of each particle. The experimental results show that this method can describe the microphysical characteristics of precipitation particles more completely and can be used to test the signal processing module of dual-polarization Doppler weather radar.

Keywords: Scattering characteristics, Marshal-Palmer distribution, Radar resolution volume, Echo signals
\end{abstract}

\section{Introduction}

The signal receiving and processing part is one of the core parts of the Doppler weather radar system, and its technical indicators play an extremely significant role in the performance evaluation of the radar. In the process of production and research and development of the weather radar, the evaluation of the signal processing performance of radar receiver is an indispensable link. In this process, it is essential to provide an effective signal source for weather radar. If we can simulate the echo sequence received by the real weather radar, not only can it optimize the design parameters of the radar system, but also help to improve the performance and advance the research and development efficiency of the weather radar. Therefore, how to quickly obtain an effective test signal source has become an important research topic.

Capsoni and D'Amico [1] proposed a multi-parameter radar simulator based on the physical characteristics of rainfall, but did not generate radar echo signals with

(c) The Author(s). 2020 Open Access This article is licensed under a Creative Commons Attribution 4.0 International License, which permits use, sharing, adaptation, distribution and reproduction in any medium or format, as long as you give appropriate credit to the original author(s) and the source, provide a link to the Creative Commons licence, and indicate if changes were made. The images or other third party material in this article are included in the article's Creative Commons licence, unless indicated otherwise in a credit line to the material. If material is not included in the article's Creative Commons licence and your intended use is not permitted by statutory regulation or exceeds the permitted use, you will need to obtain permission directly from the copyright holder. To view a copy of this licence, visit http://creativecommons.org/licenses/by/4.0/. 
polarization information. Capsoni et al. [2] simulated the multi-parameter polarization radar by setting raindrop particle distribution units with different sizes and shapes. The contribution of each particle to the radar echo is accurately estimated, but the method is lack of real-time. Cheong et al. [3] used the Advanced Regional Prediction System (ARPS) to simulate and generate an atmospheric field for avoiding the shortcomings of the Capsoni research methods, and designed and implemented a real-time radar simulator, which can generate three-dimensional time series. The atmospheric field consists of many small particles, each of which corresponds to a vector echo signal, and the radar echoes are presented by superposition of these vector signals. But this simulation method only provides the spectral moments.

Lupidi et al. [4] simulated the echoes of X-band polarization weather radar under rain and hail, and calculated the electromagnetic scattering field by utilizing the transmission matrix T-Matrix. Lischi et al. [5] designed a full polarized Doppler weather radar simulator that can generate the original time series, whose scattering and propagation effects of electromagnetic waves between particles are taken into account. The real weather radar observation data, combined with the power spectrum and propagation characteristics of the radar echo, were employed to produce the radar echo signals. Lischi et al. [6] combined the Weather Research and Forecasting (WRF) model and the transfer matrix coding to generate radar echo signals, and the 3D scenes of graupel, rain, and hail are modeled. The echo signals are gained through simulating the weather radar antenna scanning mode, and the consistent result is obtained by comparing with the real radar test data. However, in this framework, the microphysical model does not output the parameters such as oblateness and descent rate, and there are some differences between the observed results and the simulated moisture variables, especially for ice particles. Tang et al. [7] implemented a weather radar echo simulation system based on power spectrum model which takes the real radar data as the input. The simulated echo map of weather radar is produced according to the generated I and Q signals, but the algorithm limits the range of input velocity. Based on the time autocorrelation between the transmitted pulses of weather radar, Zhi-Chao et al. [8] proposed a time-domain signal simulation algorithm of weather radar under the atmospheric stratification mode, and the accuracy of the simulation algorithm was verified by taking real weak precipitation echo data of C-band dualpolarization weather radar as comparative data.

Providing weather radar echo signals with polarization information are particularly important for the research and development and testing on dual-polarization Doppler weather radar. However, the existing simulation methods require the foundation of real weather radar data and a great amount of computation. This paper, in the light of related research, applies the scattering characteristics of precipitation particles to simulate the echo of dual-polarization Doppler weather radar. This method realizes the modeling of meteorological scene based on the theoretical basis that the falling drops are not spherical but have an oblate spheroidal shape at the atmosphere. The electromagnetic scattering matrix of raindrop particles is calculated by T-matrix method, and the radar echo simulation of different polarization waves is achieved. In this paper, this method is applied to the simulation of dual-polarization Doppler weather radar echo signals. It is shown that the simulation results can more completely describe the microphysical characteristics of precipitation particles and can be employed in the test of dualpolarization Doppler weather radar signal processing module. 


\section{Methods}

\subsection{Simulation principle of echo signal of dual-polarization weather radar}

\subsubsection{The shape of raindrops}

The rainfall measurement of dual-polarization weather radar is based on the basis that the raindrops in the process of falling are not spherical but have an oblate spheroidal shape, and the shape is determined by the equivalent diameter and axis ratio. The axis ratio $b / a$, the ratio of the major axis length to minor axis length, is related to the diameter $D_{e}$ of an equivalent-volume spherical raindrop as [9]

$$
D_{e}=2\left\{\left(T_{s} / g \rho_{w}\right)\left[(b / a)^{2}-2(b / a)^{1 / 3}+1\right](b / a)^{1 / 3}\right\}^{1 / 2}
$$

where $T_{s}$ is the surface tension of water, $\mathrm{g}$ is the acceleration due to gravity, and $\rho_{w}$ is the water density.

\subsubsection{Raindrop spectrum}

Raindrop spectrum represents the distribution of the number of raindrops in the unit scale interval and per unit volume. The microphysical structure and evolution properties of precipitation can be derived by analyzing the raindrop spectrum. MarshallPalmer (M-P) distribution function is widely applied to describe the raindrop spectrum, which is given by [10]

$$
\begin{aligned}
N(D) & =N_{0} \exp (-\Lambda D) \\
\Lambda & =4.1 R^{-0.21} \\
N_{0} & =8 \times 10^{3}
\end{aligned}
$$

where $R$ is the rain rate in millimeters per hour, $D$ is the diameter of different raindrops in $\mathrm{mm}$, and $N_{0}$ is in $\mathrm{m}^{-3} \mathrm{~mm}^{-1}$.

\subsubsection{Radar meteorological equation}

Radar meteorological equation is a relationship between radar operating range, radar parameters, backscattering cross section and average echo power, which is defined as

$$
P_{r}=\frac{P_{t} g^{2} \theta \phi c \tau \pi^{3}\left|K_{w}\right|^{2} \eta_{h, v}}{512(2 \ln 2) \lambda^{2} r^{2}}
$$

where $K_{w}=\left(\varepsilon_{r}-1\right) /\left(\varepsilon_{r}+2\right)$, $\varepsilon_{r}$ is the relative dielectric constant, $P_{t}$ is the transmit power, and $g$ and $\lambda$ are the antenna gain and the wavelength of electromagnetic wave emitted by radar. $r, \theta$, and $\phi$ are distance, azimuth, and elevation, respectively, and $c$ and $\tau$ are the speed of light and the pulse width. The reflectivity $\eta_{h, v}$ of horizontal or vertical polarization, a function of the drop-size distribution, can be expressed in terms of $D_{e}$.

$$
\eta_{h, v}=\int \sigma_{h, v}\left(D_{e}\right) N\left(D_{e}\right) d D_{e}
$$

where $N\left(D_{e}\right)$ is the number of raindrop particles, and $\sigma_{h, v}$ is the backscattering cross sections of horizontal $h$ and vertical $v$ polarization. For an oblate spheroidal drop, the cross sections for horizontal and vertical incident fields are given by [11]

$$
\sigma_{h, v}=\frac{\pi^{5} D_{e}^{6}}{9 \lambda^{4}}\left|\frac{m^{2}-1}{1+\left(m^{2}-1\right) A_{h, v}}\right|^{2}
$$




$$
A_{v}=1 / e^{2}\left(1-\left(\left(1-e^{2}\right) / e^{2}\right)^{(1 / 2)} \operatorname{asin}(e)\right)=1-2 A_{h}
$$

where $m$ is the refractive index of water, and $e$ is the eccentricity of the ellipsoid,

$$
e=\left\{1-(a / b)^{2}\right\}^{1 / 2}
$$

\subsection{Radar parameters}

\subsubsection{The reflectivity estimation}

Using the meteorological equation, the reflectivity can be estimated by

$$
\hat{Z}_{h, v}=\widehat{\bar{P}}_{r e c(h, v)} \cdot \frac{1024 R_{\text {range }}^{2} \ln 2 \lambda^{2}}{P_{t} G_{t} G_{r} c \tau \theta_{1}^{2} \pi^{3}\left|K_{w}\right|^{2}}
$$

where $R_{\text {range }}$ is the radial distance, $\theta_{1}$ is the $3 \mathrm{~dB}$ beam width on the one-way power pattern, and $G_{t}$ and $G_{r}$ are the radar transmit and receive gain, respectively. The $\widehat{\bar{P}}_{\text {rec }}$ is the average power of the meteorological echo signal at the antenna, which is related to the coherent electric field echo, the radar echo electric field is given by

$$
\begin{aligned}
& \sum E_{\text {weight }} \cdot \stackrel{\mathrm{r}}{a}=\sqrt{\frac{P_{t}}{(4 \pi)^{3}}} \cdot G \lambda \sum_{n=1}^{N}\left(\frac{2 \sqrt{\pi \sigma_{n}} e^{j \psi_{n}} \cdot e^{\left(-\frac{\theta_{n}^{2}}{\gamma^{2}}-\frac{\phi_{n}^{2}}{\delta^{2}}\right)}}{r_{n}^{2}}\right) \cdot \stackrel{\mathrm{r}}{a} \\
& \gamma^{2}=\frac{\theta_{3 d B}^{2}}{4 \ln 2} \\
& \delta^{2}=\frac{\phi_{3 d B}^{2}}{4 \ln 2}
\end{aligned}
$$

Here, $\psi_{n}=2 k g_{0 n}, g_{0 n}=x_{0 n} \sin \theta \cos \phi+y_{0 n} \sin \theta \sin \phi+z_{0 n} \cos \theta$, and $x_{0 n}, y_{0 n}$ and $z_{0 n}$, are the coordinate positions of the raindrop scattering particles on the rectangular coordinate system. The $\phi$ and $\theta$ are the elevation of the particle position and the azimuth of the particle position, respectively. The $G$ is the gain of radar during transmitting and receiving, that is $G_{t}=G_{r}=G$, and the $a^{\perp}$ represents the direction of the electromagnetic scattering field.

\subsubsection{The horizontal and vertical reflectivity factor}

Some polarization parameters can be gained directly from inversion of raindrop spectrum. When the rotation axis of raindrop particles is vertically oriented, the horizontal and vertical reflectivity factors can be expressed by $\eta_{h, v}$ as:

$$
Z_{h, v}=\frac{\eta_{h, v} \lambda^{4}\left|K_{w}\right|^{2}}{\pi^{5}}
$$

\subsubsection{The differential reflectivity factor}

The differential reflectivity factor $Z_{D R}$ is the logarithm of the ratio of horizontal reflectivity factor $Z_{h}$ to vertical reflectivity factor $Z_{v}$, which reflects the intensity difference between the two polarization directions, and can be obtained by using the horizontal and vertical reflectivity factors. 


$$
Z_{D R}=10 \log \left(\frac{Z_{h}}{Z_{v}}\right)
$$

The unit of $Z_{D R}$ is $d B$, which has nothing to do with the number distribution of precipitation particles in the radar resolution volume, but is related to the shape distribution of precipitation particles. It reflects the spatial orientation of precipitation particles deviated from the sphere and particle swarm.

\subsubsection{The specific differential phase}

Raindrop particles will cause phase shift to electromagnetic waves. Due to the resistance of air, and the long axis of oblate spheroidal raindrops is horizontal, the phase shift of horizontal electric field is relatively larger. The difference between the phase change of horizontal and vertical wave propagation is represented by the specific differential phase $\left(K_{D P}\right)$, which is not affected by beam blocking or ground clutter and is a little affected by attenuation, so the application of $K_{D P}$ in quantitative precipitation measurement can improve the precision. The $K_{D P}$ is usually related to the shape, dielectric constant, and numerical density of moisture variables, it can be expressed as

$$
K_{D P}=\frac{180 \lambda}{\pi} \operatorname{Re}\left\{\int_{0}^{D_{m}}\left[f_{h}\left(D_{e}\right)-f_{v}\left(D_{e}\right)\right] N\left(D_{e}\right) d D_{e}\right\}
$$

where $f_{h}$ and $f_{v}$ are horizontal and vertical scattering coefficients, respectively.

\subsubsection{The average radial velocity}

The average radial velocity can be given by

$$
\widehat{\bar{v}}=\frac{1}{N-1} \frac{\lambda f_{d}}{4 \pi} \sum_{n=1}^{N-1} \arg \left\{V_{n+1} V_{n}^{*}\right\}
$$

where $V_{n}$ is radial velocity, $f_{d}$ is Doppler shift, and $N$ is the number of pulses.

\subsection{Simulation model of polarization radar echo signal}

\subsubsection{Radar resolution volume}

The pulse signal beam emitted by radar can be regarded as conical, and the radar resolution volume is a segment of the volume of the cone, which is drawn in Fig. 1.

The volume size of radar resolution is calculated as

$$
V_{\text {res }}=\frac{\pi}{4} \cdot\left(r_{i} \theta_{3 d B}\right)\left(r_{i} \phi_{3 d B}\right)\left(\frac{c \tau}{2}\right)
$$

The initial parameters of the distribution position of raindrop particles are set within the resolution volume. Because of the motion of raindrop particles, it is necessary to set the boundary range of the resolution volume to determine whether the moving raindrop particles are still within the resolution volume. For the convenience of calculation, the position of raindrops is defined by polar coordinates.

\subsubsection{The matrix of raindrop particle size distribution}

The T-matrix can be constructed by raindrop parameters, which are listed in Table 1.

Using Eqs. (2) and (5), we can calculate the required data and write them into a matrix, that is, the T-matrix mentioned above is given by 


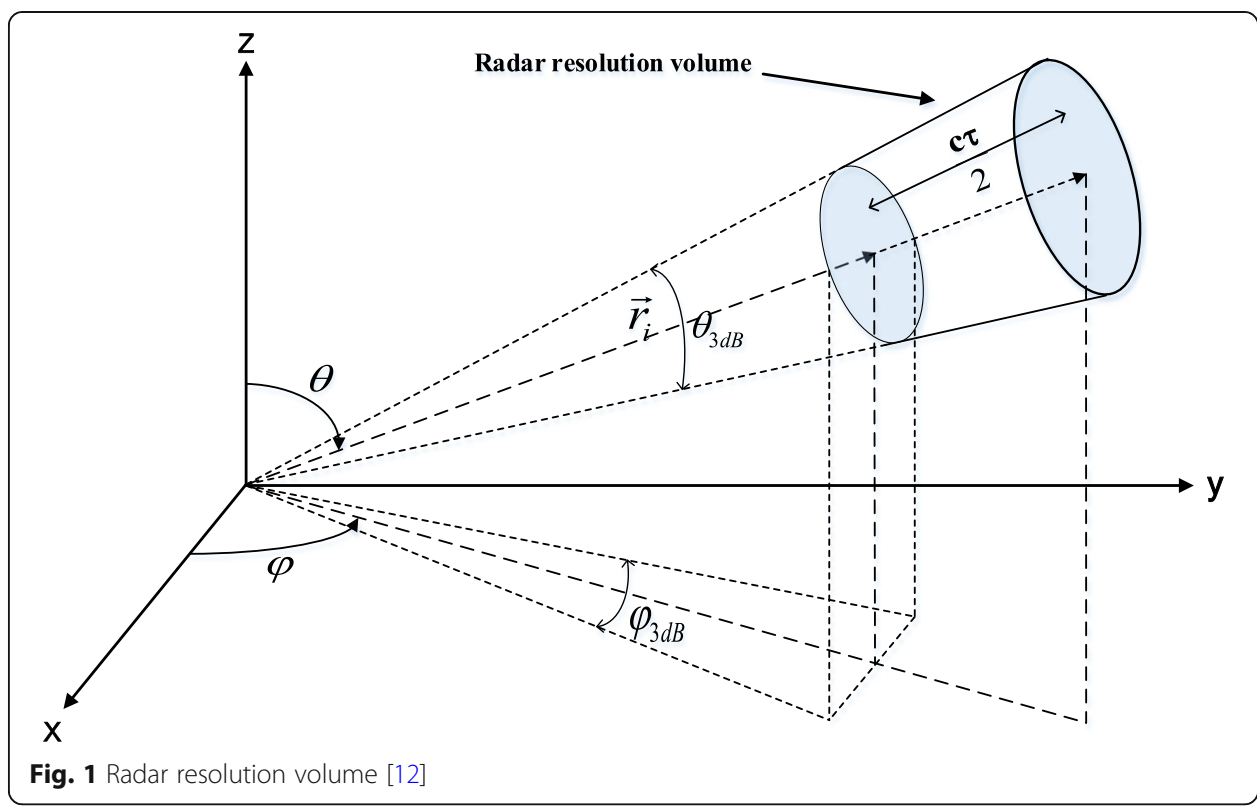

$$
\text { matrix_T_round }=\left[\begin{array}{ccc}
D_{e 1} & \ldots & D_{e N} \\
\sigma_{1 v} & \ldots & \sigma_{N v} \\
\sigma_{1 h} & \ldots & \sigma_{N v} \\
N\left(D_{1}\right) & \ldots & \mathrm{N}\left(D_{N}\right)
\end{array}\right]
$$

\subsubsection{The matrix of raindrop position}

The position of each raindrop particle in the resolution volume is randomly distributed. The position information is described by the spherical coordinate system and stored in a raindrop position matrix, which can be written as

$$
\text { matrixpos }=\left[\begin{array}{llc}
r_{1} & \ldots & r_{N} \\
\phi_{1} & \ldots & \phi_{N} \\
\theta_{1} & \ldots & \theta_{N} \\
\text { angle }_{1} & \ldots & \text { angle }_{N}
\end{array}\right]
$$

where $r$ represents the radial distance from the radar station, $\phi$ is the elevation of the particle position, and $\theta$ is the azimuth of the particle position. The angle is the angle

Table 1 The raindrop particle parameters

\begin{tabular}{ll}
\hline Parameter & Value \\
\hline Minimum particle diameter $(\mathrm{mm})$ & 0.01 \\
Boundary diameter of small and medium particles $(\mathrm{mm})$ & 0.5 \\
Boundary diameter of large and medium particles $(\mathrm{mm})$ & 3 \\
Maximum particle diameter $(\mathrm{mm})$ & 6 \\
Low size particle resolution $(\mathrm{mm})$ & 0.03 \\
Medium size particle resolution $(\mathrm{mm})$ & 0.1 \\
High size particle resolution $(\mathrm{mm})$ & 0.3 \\
Wind velocity vector $(\mathrm{m} / \mathrm{s})$ & {$[-5,0,0]$}
\end{tabular}


between the particle and the centerline of the beam, which is used to determine which raindrop particles are inside the beam.

For each raindrop of diameter size, the raindrop within the resolution volume is selected according to the Eq. (18). The position of the raindrop is updated by applying the wind velocity vector and the terminal velocity, which can be expressed as

$$
v(D) \approx 386.6 D^{0.67}
$$

where $D$ is the particle diameter in $\mathrm{m}$, and the unit of $v$ is meters/second. Using the pulse repetition time, we can determine the moving distance of the raindrop particles, and the raindrop particle position corresponding to each pulse time is saved in the position matrix.

\subsubsection{Polarization radar echo simulator}

According to the particle scattering model and the detection principle of dualpolarization weather radar, a dual-polarization weather radar echo simulator can be constructed. The input parameters of the simulator mainly include two parts, namely, radar parameters and weather parameters, as shown in Table 2. The output of the simulator is the radar echo signals of vertical polarization and horizontal polarization.

The simulator uses different size resolutions to determine the equivalent diameter of particles in different size ranges according to the size range of raindrops. In the experiment, the axis ratio $b / a$ needs to be computed from the size of the equivalent diameter, but it is difficult to solve Eq. (1) directly. Hence, the least square algorithm is introduced to find the best match of the data by calculating the square of the error minimized. The distribution curves of axis ratio and equivalent diameter are fitted by the least square algorithm, and the resolution of the equivalent diameter of the original data points is $0.01 \mathrm{~cm}$, which can ensure that the corresponding axis ratio can be obtained by setting different precision and range of equivalent diameter (Table 1).

Firstly, the distribution of the original data points is drawn, and then the least square algorithm is used to fit the original discrete data points according to the distribution. The fitting effect is shown in Fig. 2, it can be seen clearly that the fitting result of the least square algorithm is consistent with the theoretical data.

Table 2 Model input parameters

\begin{tabular}{ll}
\hline Model input parameter & Meaning \\
\hline$P_{t}(\mathrm{~kW})$ & Transmitted signal power \\
$G(\mathrm{~dB})$ & Transmit and receive antenna gain \\
$r(\mathrm{~km})$ & Distance of resolution volume \\
$\theta\left(^{\circ}\right)$ & Beam width \\
$T(\mathrm{us})$ & Pulse width \\
$\lambda(\mathrm{cm})$ & Wavelength \\
$T(\mathrm{~s})$ & Pulse interval \\
$N(D)$ & Raindrop spectrum parameters \\
$D(\mathrm{~mm})$ & Raindrop resolution size \\
$V(\mathrm{~m} / \mathrm{s})$ & Average velocity and direction of Raindrop \\
$(r, \varphi, \theta)$ & Raindrop position \\
\hline
\end{tabular}


Thus, the effective scattering area of each raindrop particle can be calculated according to Eq. (5).

When the rain rate and particle size are determined, the number concentration $N(D)$ corresponding to particles of different sizes can be gained by raindrop spectrum distribution function Eq. (2).

The radar effective scattering cross section can be obtained by the vector superposition of the scattering echo of each raindrop particle, which is given by

$$
E_{\text {total }}\left(t_{k}\right)=\sum_{s} \sum_{i=1}^{N_{i}} \sqrt{\sigma_{D_{s}}} \cdot e^{i 2 k} \vec{r}_{i}\left(t_{k}\right)
$$

where $t_{k}$ is the sampling time and $s$ is the diameter of raindrop particles.

The echo simulator finally outputs the weighted electric field echo, which can be applied to calculate the precipitation parameters such as echo power, reflectivity, differential reflectivity, and velocity.

\section{Results and discussions}

In this study, the technical indicators of dual-polarization Doppler weather radar can be set according to the need. In the precipitation radar echo simulation experiment, it is assumed that the precipitation raindrops are not electrified, and uncharged water drops commonly do not unite on collide but rebound, so there is no uniting and loss of raindrops. And the main technical indicators of the dual-polarization Doppler weather radar used are presented in Table 3.

In order to prove the accuracy of the simulation signal, the time-frequency characteristics of the signal are analyzed, and the results are as follows. The form of echo voltage is shown in Fig. 3. It is obvious that the variation rules of horizontal and vertical echo voltage are basically coincident.

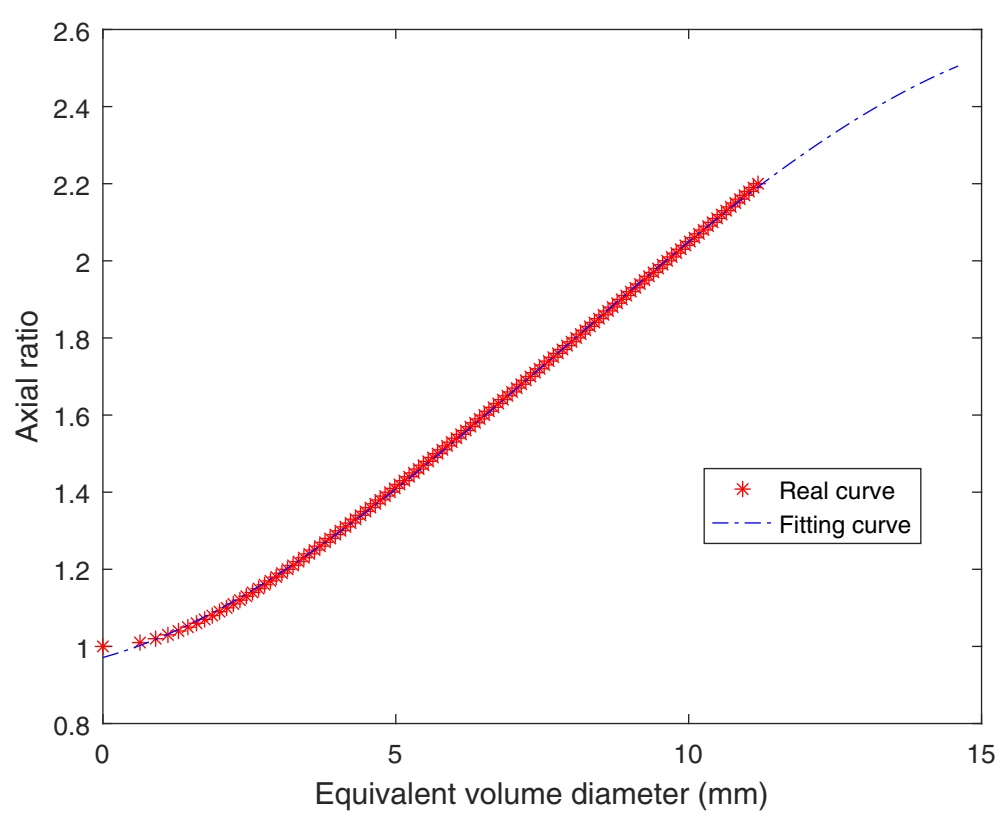

Fig. 2 Fitting effect of the least square method 
The meteorological echo is composed of a large number of discrete scattered particle echoes, the position of these scattered particles will change due to the movement of the scatterer, and the amplitude of the echo will fluctuate in that the change of the position. Therefore, the statistical characteristics of echo amplitude can reflect part of the particle variation information. Figure 4 shows the statistical characteristics of echo amplitude in time-domain. It can be seen that the echo amplitude spectrum has the Rayleigh distribution, which is determined by the microphysical characteristics of precipitation itself. The statistical characteristics of the phase distribution are shown in Fig. 5, from where we can see that the phase has the uniform distribution, which is essentially in agreement with the statistical characteristics of precipitation echoes described in reference [13].

The meteorological echo signal is a random fluctuation signal, and its power spectrum can be represented by power spectral density, as shown in Fig. 6. Figure 6a shows the normalized power spectral density for echo of horizontally polarized waves, which is calculated by FFT (fast Fourier transforms) algorithm and Fourier transform of autocorrelation algorithm, respectively. The corresponding power spectral density of vertically polarized waves is shown in Fig. 6b, from which we can conclude that the distribution of power spectral density calculated by these two algorithms is basically the same for both vertical polarization and horizontal polarization. And the distribution regulation is approximate to Gaussian distribution, which conforms to the conclusion of reference [14].

Figure 7 shows the histogram of distribution of the radial velocity calculated according to each pulse pair, and the longitudinal coordinate represents the frequency of the velocity interval. The Gaussian fitting function is used for fitting the histogram. It is clear that the transverse coordinate value corresponding to the peak value of the Gaussian function is essentially consistent with the Doppler frequency shift corresponding to the peak value of the power spectrum, and the previous is fundamentally same with the initial set velocity value.

Figure 8 shows the reflectivity of each pulse when it returns. The reflectivity of each pulse is estimated by receiving voltage, and the average value of each pulse is calculated, as shown in the average reflectivity curve in Fig. 8, where the actual reflectivity is calculated according to the distribution function of droplet size. It can be seen that the changing trends of the two polarization directions are basically the same, and the reflectivity of horizontal polarization is about $2 \mathrm{dBZ}$ larger than that of vertical polarization, which is identical with that described in reference [15]. And it can be concluded that the reflectivity calculated by the droplet size distribution function is slightly

Table 3 The main technical indicators of the radar used in the experiment

\begin{tabular}{lll}
\hline Number & Project & Value \\
\hline 1 & Transmitted signal power (kW) & 60 \\
2 & Antenna gain (dB) & 20 \\
3 & Beam width $\left(^{\circ}\right)$ & 1 \\
4 & Pulse width (us) & 1 \\
5 & Wavelength (cm) & 10 \\
6 & Pulse repetition time (s) & 0.002 \\
\hline
\end{tabular}




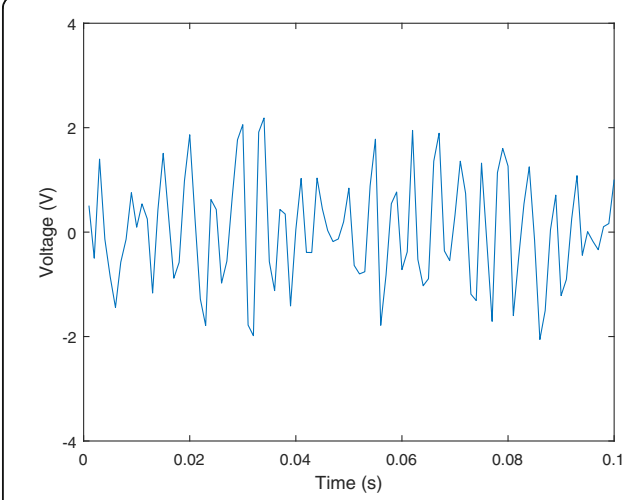

(a)

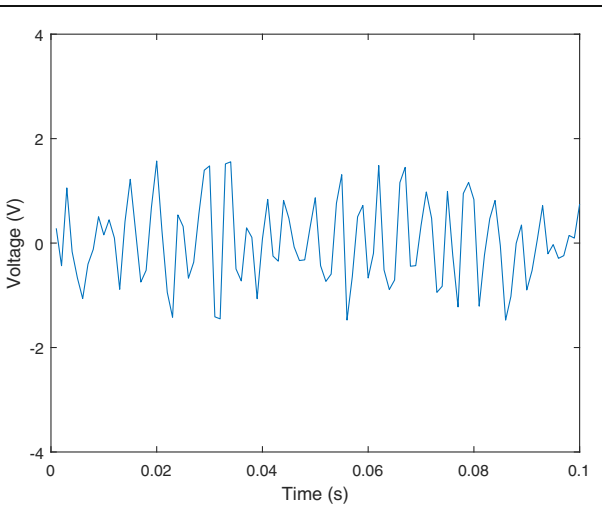

(b)

Fig. 3 Echo voltage. a Horizontal polarization echo voltage. b Vertical polarization echo voltage

larger than the estimated reflectivity, which proves that the simulation results can accord with the theoretical values.

In order to verify the accuracy of the precipitation model, the value of $K_{D P}$ is calculated by Eq. (14) in the light of the distribution of raindrop size, as shown in Fig. 9. It is obvious from Fig. 9 that the value of $K_{D P}$ increases with the increase of rain rate, the reason is that when the rain rate is large, the raindrop distribution function assumed by the model corresponds to more heavy raindrop particles, while $K_{D P}$ is related to raindrop size distribution [16].

By setting different rain rates, the relationship with the reflectivity can be derived, as shown in Fig. 10. The red dots represent the reflectivity factor calculated by the horizontal echo data, and the green dots indicate the reflectivity factor calculated by the vertical echo data. The least square algorithm is applied to fit the horizontal and vertical reflectivity factor data to acquire the relationship between reflectivity factor and rain

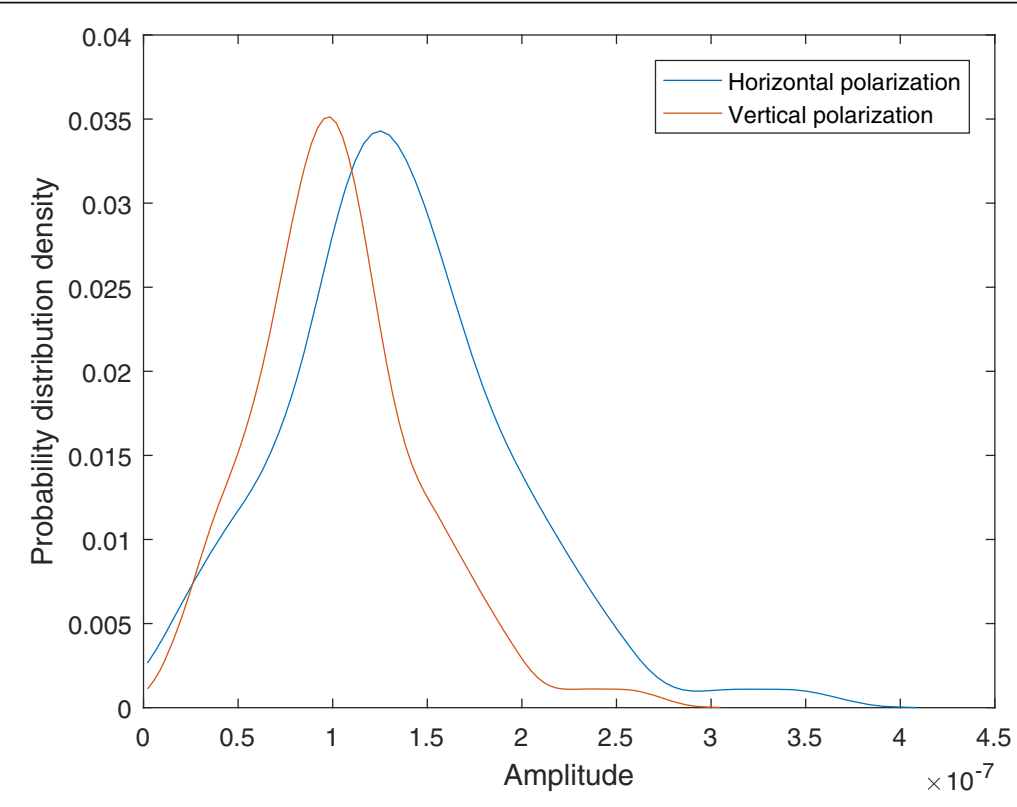

Fig. 4 Amplitude probability distribution density 


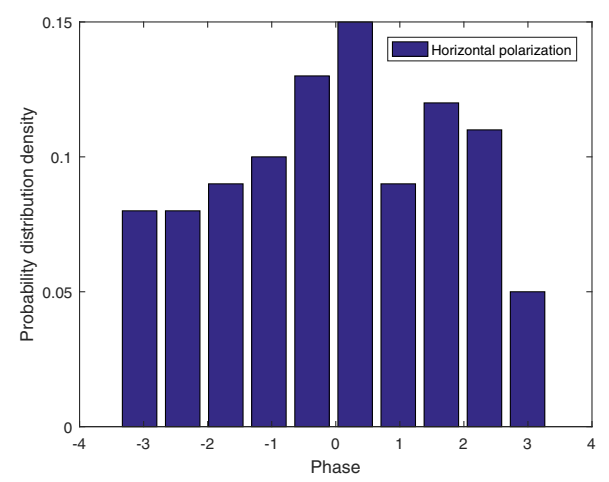

(a)

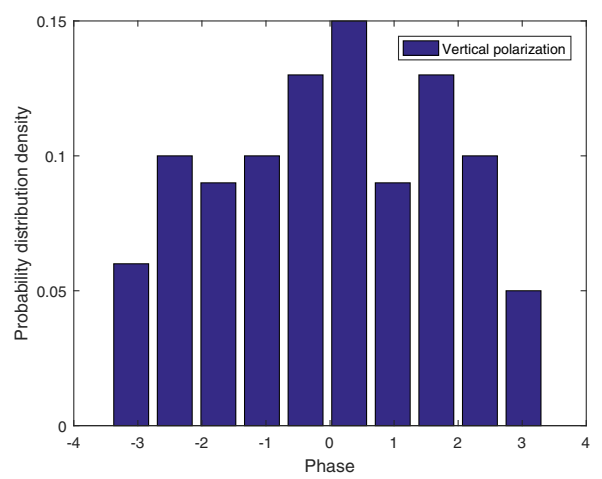

(b)

Fig. 5 Phase probability distribution density. a Horizontal polarization. b Vertical polarization

rate, and the fitting curve effect as shown in Fig. 10. The theoretical curves $Z_{h}, R$ and $Z_{v}, R$ relationship in the figure come from Crozier et al. [15]. It can be seen that the horizontal reflectivity factor is slightly larger than the vertical reflectivity factor, and its distribution law basically conforms to the theoretical research results of Crozier et al.

\section{Conclusions}

In this paper, the simulation of precipitation echo of dual-polarization weather radar is realized by using the theoretical basis that the falling drops are not spherical but have an oblate spheroidal shape at the atmosphere. The Marshall-Palmer distribution is used to describe the raindrop spectrum distribution of precipitation particles, and it is assumed that the raindrop particles have random distribution in the radar resolution volume; thus, the spatial distribution of precipitation particles in the radar resolution volume is modeled. The least square algorithm is applied to solve the problem that it is difficult to calculate the axis ratio of precipitation particles. The backscattering echo of each precipitation particle under horizontal and vertical polarization electromagnetic wave is calculated by simulating the dual-polarization radar detection mechanism, and then the echo signals of dual-polarization radar in horizontal and vertical polarization channels are obtained by vector superposition of the echo signals of precipitation

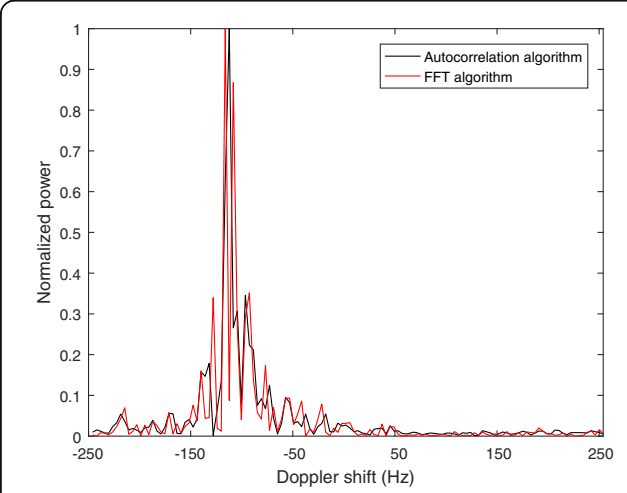

(a)

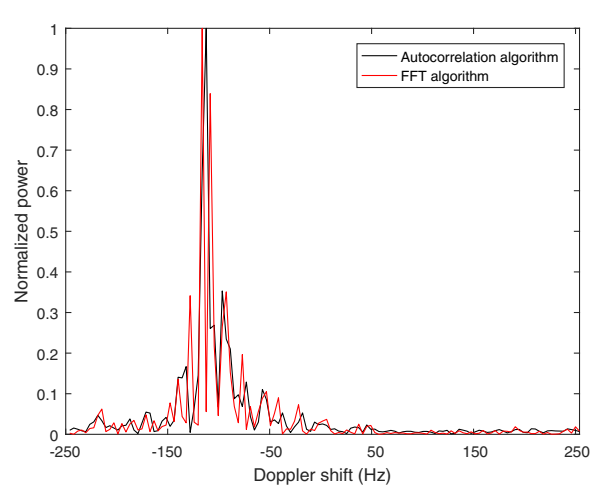

(b)

Fig. 6 Power spectrum. a Horizontal polarization power spectrum. b Vertical polarization power spectrum 


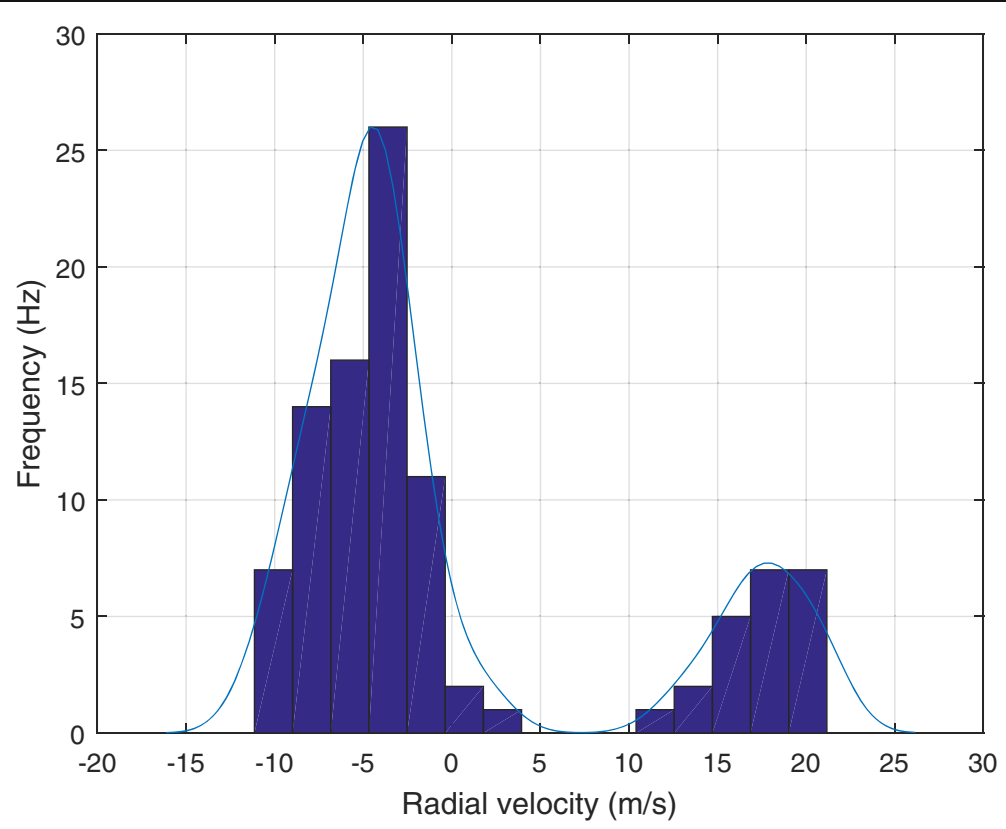

Fig. 7 Radial velocity distribution

particles. The experimental results show that this method can describe the microphysical characteristics of precipitation particles more completely and can be used in the test of dual-polarization Doppler weather radar signal processing module. It is of great significance to the research and development of dual-polarization weather radar. Future studies can generate echo map according to the echo signals obtained by this paper and simulate the radar echoes of different precipitation phenomena.

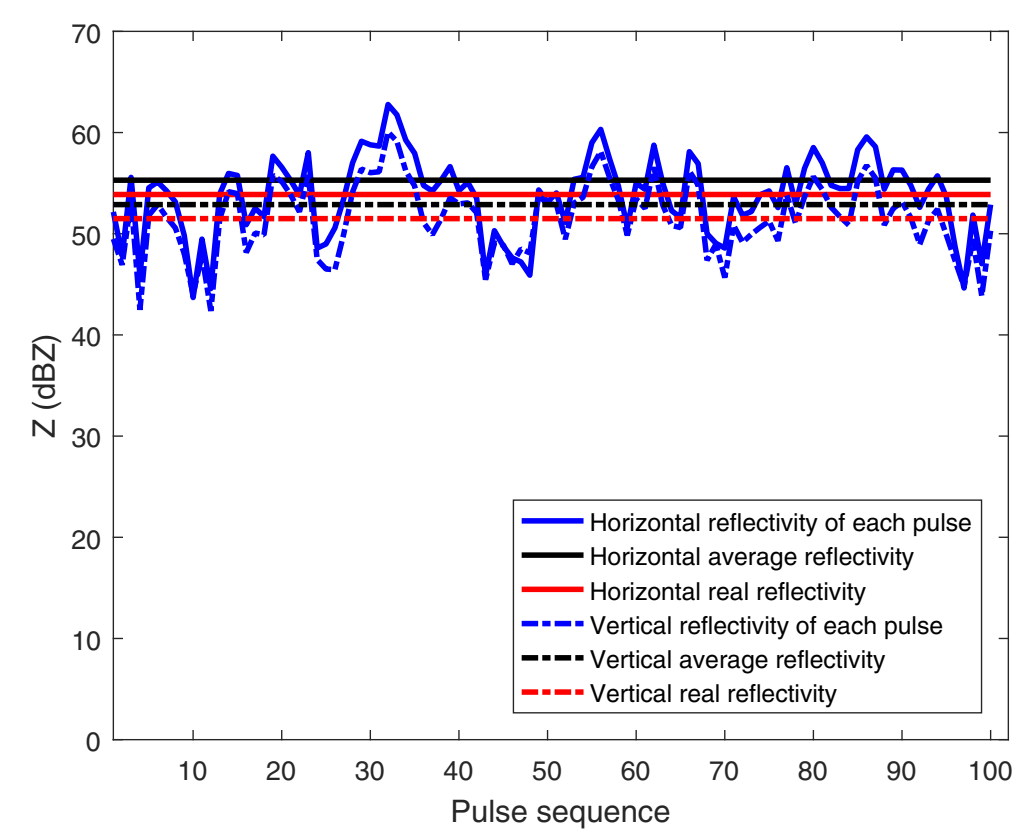

Fig. 8 Reflectivity estimation 


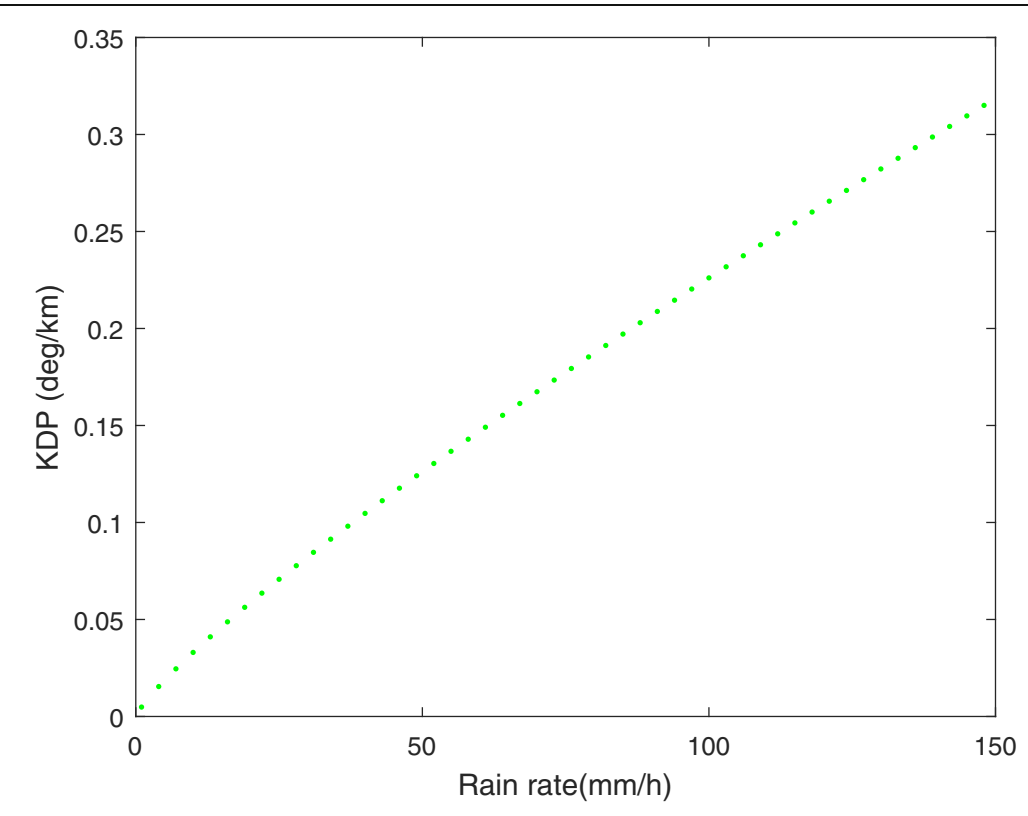

Fig. 9 Relationship between specific differential phase and rain rate

The real atmospheric environment is complex, and the propagation speed and energy loss of electromagnetic waves are different in different meteorological environments, so the loss of electromagnetic waves in the propagation process is not considered in the experiment. These factors have a certain impact on radar echo simulation modeling, which can be taken into account in the follow-up research, so there is still room for improvement in the method.

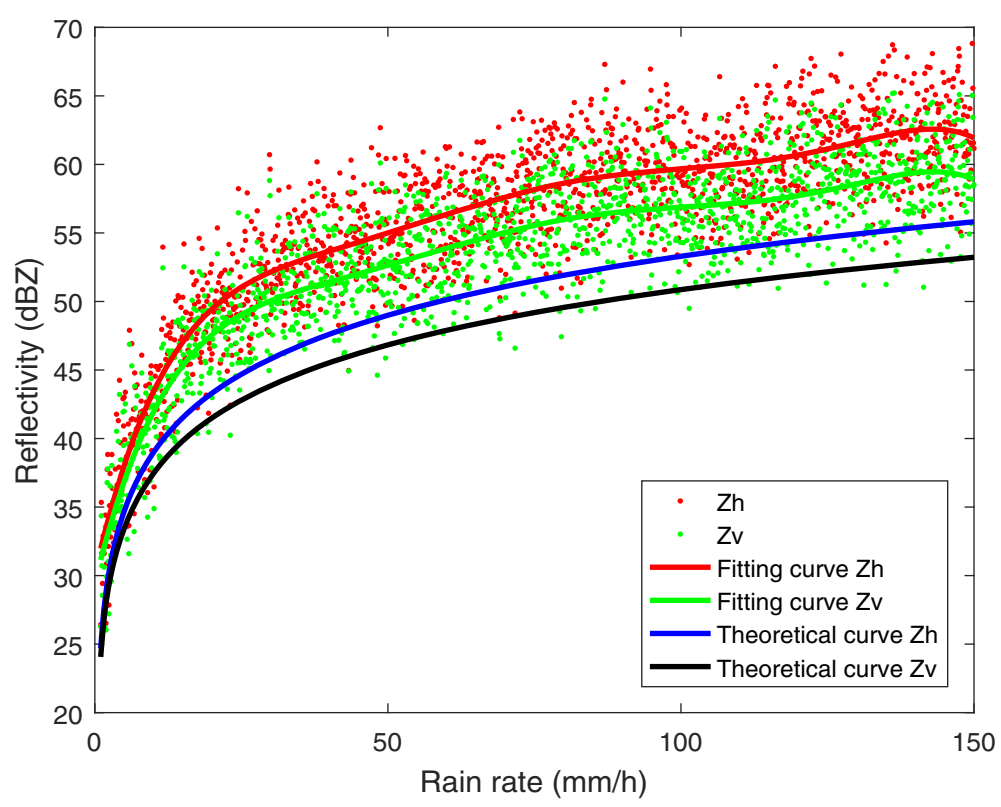

Fig. 10 Relationship between rain rate and reflectivity 


\section{Abbreviations}

M-P: Marshal-Palmer; ARPS: The Advanced Regional Prediction System; WRF: The Weather Research and Forecasting; FFT: The fast Fourier transforms

\section{Authors' contributions}

HJW proposed the study and put forward the echo simulation model. QTJL conducted the experiments and wrote the manuscript. SPH and JHZ analyzed and evaluated the experimental results. MQG drew the figures and improved the structure of the manuscript. All authors read and approved the final manuscript.

\section{Funding}

This research was funded by the National Natural Science Foundation of China (Joint Fund of Civil Aviation Research, award number U1733103).

\section{Availability of data and materials}

Data sharing is not applicable to this article as no datasets were generated or analyzed during the current study.

\section{Competing interests}

The authors declare that they have no competing interests.

Received: 16 July 2020 Accepted: 29 September 2020

Published online: 12 October 2020

\section{References}

1. C. Capsoni, M. D'Amico, A physically based radar simulator[J]. J. Atmos. Ocean. Technol. 15(2), 593-598 (1998)

2. C. Capsoni, M. D'Amico, R. Nebuloni, A multiparameter polarimetric radar simulator[J]. J. Atmos. Ocean. Technol. 18(11), 1799-1809 (2001)

3. B.L. Cheong, R.D. Palmer, M. Xue, A time series weather radar simulator based on high-resolution atmospheric models[J]. J. Atmos. Ocean. Technol. 25(2), 230-243 (2008)

4. Lupidi A, Moscardini C, Berizzi F, et al. Simulation of X-band polarimetric weather radar returns based on the Weather Research and Forecast model[C] Radar Conference (RADAR), 2011 IEEE. IEEE, 2011: 734-739.

5. S. Lischi, A. Lupidi, F. Berizzi, et al., X-band full polarized Doppler weather radar return simulation by using propagationmodified ensemble-averaged covariance matrix[C] Radar Symposium (IRS), 2013 14th International. IEEE 2, 799-804 (2013)

6. Lischi S, Lupidi A, Martorella M, et al. Advanced polarimetric Doppler weather radar simulator[C]//Radar Symposium (IRS), 2014 15th International. IEEE, 2014: 1-6

7. Tang Shunxian, Li Rui, Shi Zhao, et al. Design and implementation of the echo signals simulation system of weather radar [J]. 2014,40(11):136-138.

8. Zhi-Chao B U, Nan S, Bai L I, et al. The simulation model and statistical validation of I/Q signal for weather radar[J]. ence Technology and Engineering, 2017.

9. A.W. Green, An approximation for the shapes of large raindrops[J]. J. Appl. Meteorol. 14(8), 1578-1583 (1975)

10. J.S. Marshall, W. Palmer, The distribution of raindrops with size. J. Meteorol. 5, 165-166 (1948)

11. D.P. Stapor, T. Pratt, A generalized analysis of dual-polarization radar measurements of rain[J]. Radio Sci. 19(1), 90-98 (2016)

12. Zhang Peichang. Radar meteorology [M]. Meteorological Publishing House, 1988.

13. Doviak, Richard, Zrnic, S. Doppler radar and weather observations[M]// Doppler radar and weather observations. Dover Publications, 2006

14. M. Pinsky, F.I.V. Jordi, T. Otto, et al., Application of a simple adaptive estimator for an atmospheric Doppler radar[J]. IEEE Trans. Geosci. Remote Sens. 49(1), 115-127 (2011)

15. Crozier, C. L., Joe, P. I., Scott, J. W., Herscovitch, H. N., Nichols, T. R. First experiment with an operational Doppler radar. Prepr., Radar Meteorol. Conf., 24th, 1989. 1989; 179-185.

16. T. Oguchi, Electromagnetic wave propagation and scattering in rain and other hydrometeors[J]. Proc. IEEE 71(9), 10291078 (1983)

\section{Publisher's Note}

Springer Nature remains neutral with regard to jurisdictional claims in published maps and institutional affiliations. 